\title{
Subject Index to Volume 21 - 1994
}

Abstracts

Canadian Association of Neuropathologists 159 (May)

Canadian Congress of Neurological Sciences (Suppl. 2)

Adaptation

Adaptive Control of Eye Movements: Clinical Implications 177 (Aug)

Alcoholism

Alcoholic Dementia: A Critical Review 88 (May)

Alzheimer's Disease

Alzheimer's as a Common Complex Disease 78 (Feb)

The Short History of Focal Brain Degeneration 78 (Feb)

SPECT for Differential Diagnosis of Dementia and Correlation of $\mathrm{rCBF}$ with Cognitive Impairment 104 (May)

Progression and Outcome of Patients Referred to a Canadian Dementia Clinic 331 (Nov)

Anticonvulsant

The Effect of Anticonvulsant Drugs on GABAStimulated Chloride Uptake 3 (Feb)

Antiepileptic Drugs

Ataxia in Institutionalized Patients with Epilepsy 252 (Aug)

Antiepileptic Drug Selection and Adverse Effects: An Overview S3 (Suppl 3)

Acute and Chronic Toxicity of Antiepileptic Medications: a Selective Review S7 (Suppl 3)

Cognitive Side Effects of Antiepileptic Drugs S12 (Suppl 3)

How About the New Antiepileptic Drugs? S17 (Suppl 3)

Arteriovenous Malformations

Fatal Rebleeding from a Dural Arteriovenous Malformation of the Posterior Fossa: case report with pathological examination 67 (Feb)

Astrocytomas

Complications of First Craniotomy for Intra-Axial Brain Tumor 213 (Aug)

Proliferating Cell Nuclear Antigen Expression in Ataxia the Survival of Astrocytoma Patients 306 (Nov)

Ataxia in Institutionalized Patients with Epilepsy 252 (Aug)

Parieto-Cerebellar Loop Impairment in Ataxic Hemiparesis: Proposed Pathophysiology Based on an Analysis of Cerebral Blood Flow 15 (Feb)

Carotid Artery Dissection

Surgical Referrel for Carotid Artery Stenosis - The Influence of NASCET 129 (May)

Cerebellar Dysfunction

Ataxia in Institutionalized Patients with Epilepsy 252 (Aug)

Neuropsychological Functioning in Unilateral Cerebellar Damage 353 (Nov)

Cerebellar Incoordination

Parieto-Cerebellar Loop Impairment in Ataxic Hemisparesis: Proposed Pathophysiology Based on an Analysis of Cerebral Blood Flow 15 (Feb)

\section{Cerebral Blood Flow}

Parieto-Cerebellar Loop Impairment in Ataxic Hemiparesis: Proposed Pathophysiology Based on an Analysis of Cerebral Blood Flow 15 (Feb)

SPECT for Differential Diagnosis of Dementia and Correlation of $\mathrm{rCBF}$ with Cognitive Impairment I04 (May)
Overview of Monitoring of Cerebral Blood Flow and Metabolism After Severe Head Injury S6 (Suppl 1)

Cerebral Edema

Acute and Chronic Cerebral White Matter Damage in Neonatal Hydrocephalus 299 (Nov)

Cerebral Metabolism

Overview of Monitoring of Cerebral Blook Flow and Metabolism After Severe Head Injury S6 (Suppl 1)

Cerebrovascular Disease

Progress in Understanding the Role of Carotid Endarterectomy 104 (May)

SPECT for Differential Diagnosis of Dementia and Correlation of rCBF with Cognitive Impairment 104 (May)

Iatrogenic False Aneurysm Following Repair of Intracranial Aneurysm 346 (Nov)

Neuropsychological Functioning in Unilateral Cerebellar Damage 353 (Nov)

Diagnosis of Vascular Dementia: Consortium of Canadian Centres for Clinical Cognitive Research Consensus Statement 358 (Nov)

\section{Classification}

A Proposal for Classification of Neurocysticercosis 43 (Feb)

Cognitive Impairment

Cognitive Side Effects of Antiepileptic Drugs S12 (Suppl 1)

Computed Tomography

Unusual Evolution and Computerized Tomographic Appearance of a Gliosarcoma 141 (May)

Diagnosis of Vascular Dementia: Consortium of Canadian Centres for Clinical Cognitive Research Consensus Statement 358 (Nov)

Creutzfeldt-Jakob Disease

Unilateral Creutzfeldt-Jakob Disease Presenting as Rapidly Progressive Aphasia 350 (Nov)

Cysticercosis

A Proposal for Classification of Neurocysticercosis 43 (Feb)

Degenerative Diseases-Dementia

Alzheimer's as a Common Complex Disease 78 (Feb)

The Short History of Focal Brain Degeneration 78 (Feb)

Unilateral Creutzfeldt-Jacob Disease Presenting as Rapidly Progressive Aphasia 350 (Nov)

Dementia

Alzheimer's as a Common Complex Disease 78 (Feb)

The Short History of Focal Brain Degeneration 78 (Feb)

Alcoholic Dementia: A Critical Review 88 (May)

SPECT for Differential Diagnosis of Dementia and Correlation of $\mathrm{rCBF}$ with Cognitive Impairment 104 (May)

Progression and Outcome of Patients Referred to a Canadian Dementia Clinic 331 (Nov)

Unilateral Creutzfeldt-Jakob Disease Presenting as Rapidly Progressive Aphasia 350 (Nov)

Diagnosis of Vascular Dementia: Consortium of Canadian Centres for Clinical Cognitive Research Consensus Statement 358 (Nov)

Demyelination

The Impact of Fatigue on Patients with Multiple Sclerosis 9 (Feb)
Diabetes Mellitus

Complications of Sural Nerve Biopsy in Diabetic verus Non-Diabetic Patients 34 (Feb)

Clinical Testing in Diabetic Neuropathy S3 (Suppl 4)

Role of Electrophysiological Studies in Diabetic Neuropathy S8 (Suppl 4)

Pathological Definition and Evaluation of Diabetic Neuropathy and Clinical Correlations S13 (Suppl 4)

End-Stage Complications of Diabetic Neuropathy: Foot Ulceration S18 (Suppl4)

Update on Diabetic Neuropathy - Speakers' Panel S23 (Suppl 4)

Diabetic Neuropathy

The Influence of Sulindac on Experimental Streptozotocin-Induced Diabetic Neuropathy 194 (Aug)

Update on Diabetic Neuropathy - Supplement 4

Diastematomyelia

Adult Diastematomyelia 72 (Feb)

Dysgenesis

The Place of Neuronal Migration Abnormalities in Child Neurology 185 (Aug)

Dysplasias

The Place of Neuronal Migration Abnormalities in Child Neurology 184 (Aug)

Dysraphism

Adult Diastematomyelia 72 (Feb)

Adult Diastematomyelia Letter 365 (Nov)

Editorial

Progress in Understanding the Role of Carotid Endarterectomy 85 (May)

Planning Care of Neurology and Neurosurgery Patients with Critical Illnesses 295 (Nov)

Education

MOCOMP: An Idea Whose Time Has Come for Canadian Neurology 285 (Aug)

Electroencephalography

Electrophysiologic Monitoring in the Intensive Care Unit S12 (Suppl I)

Electromyography

Usefulness of Single Fiber EMG for Distinguishing Neuromuscular from other Causes of Ocular Muscle Weakness 125 (May)

Endarterectomy

Progress in Understanding the Role of Carotid Endarterectomy 85 (May)

Surgical Referral for Carotid Artery Sienosis - The Influence of NASCET 129 (May)

Epidemiology

The Impact of Fatigue on Patients with Multiple Sclerosis 9 (Feb)

Surgical Referral for Carotid Artery Stenosis - The Influence of NASCET 129 (May)

A Prevalence Study of Multiple Sclerosis in the Crowsnest Pass Region of Southern Alberta 262 (Aug)

Progression and Outcome of Patients Referred to a Canadian Dementia Clinic 33I (Nov)

Epilepsy

The Effect of Anticonvulsant Drugs on GABAStimulated Chloride Uptake 3 (Feb)

Ataxia in Institutionalized Patients with Epilepsy 252 (Aug)

Antiepileptic Drug Selection and Adverse Effects: An Overview S3 (Suppl 3)

Acute and Chronic Toxicity of Antiepileptic Medications: a Selective Review S7 (Suppl 3) Cognitive Side Effects of Antiepileptic Drugs S 12 (Suppl 3) 
Evoked Potentials

Minimum Standards for Clinical Evoked Potentials 75 (Feb)

Electrophysiologic Monitoring in the Intensive Care Unit S12 (Suppl 1)

A New Quantitative Measure for Monitoring Somatosensory Evoked Potentials S17 (Suppl 1)

Somatosensory Evoked Potential and Intracranial Pressure in Severe Head Injury 219 (Aug)

Spinal Somatosensory Evoked Potentials in Patients with Tethered Cord Syndrome 325 (Nov)

Eye Movements

Adaptive Control of Eye Movements: Clinical Implications 177 (Aug)

Fluoxetine

Fluoxetine and Selegiline - Lack of Significant Interaction 259 (Aug)

GABA

The Effect of Anticonvulsant Drugs on GABAStimulated Chloride Uptake 3 (Feb)

Genetics

Autosomal Recessive Motor and Sensory Neuropathy with Excessive Myelin Outfolding in Two Siblings 29 (Feb)

Autosomal Recessive, Fatal Infantile Hypertonic Muscular Dystrophy among Canadian Natives 203 (Aug)

Genetics - Molecular Biology

Assessment of Genetic Polymorphisms in DNA from Formalin Fixed Neurological Tissues 248 (Aug)

Gilles de la Tourette Syndrome

Gilles de la Tourette Syndrome 48 (Feb)

Glioma

Unusual Evolution and Computerized Tomographic Appearance of a Gliosarcoma 141 (May)

Complications of First Craniotomy for Intra-Axial Brain Tumor 213 (Aug)

Guillain Barré

Intensive Care of Acute Guillain-Barré Syndrome S23 (Suppl 1)

Head Trauma

Overview of Monitoring of Cerebral Blood Flow and Metabolism After Severe Head Injury S6 (Suppl 1)

A New Quantitative Measure for Monitoring Somatosensory Evoked Potentials S17 (Suppl I)

Somatosensory Evoked Potential and Intracranial Pressure in Severe Head Injury 219 (Aug)

Hemorrhage Otology

Fatal Rebleeding from a Dural Arteriovenous Malformation of the Posterior Fossa: case report with pathological examination 67 (Feb)

Hiccup

Intractable Hiccup due to Multiple Sclerosis: MR Imaging of Medullary Plaque 27 I (Aug)

Hippocampus

Hippocampal Stimulation of Fornical-lesioned History Rats Improves Working Memory 100 (May)

The Short History of Focal Brain Degeneration 78 (Feb)

Neurosurgery at the Toronto General Hospital, 1924 - 1990: Part I 146 (May)

Neurosurgery at the Toronto General Hospital 1924 - 1990: Part 2 278 (Aug)

Hydrocephalus

Acute and Chronic Cerebral White Matter Damage in Neonatal Hydrocephalus 299 (Nov)

Hypothermia

Mild Hypothermia Preserves Contractile Function and Inhibits Prostaglandin E2 Release from
Metabolically Stressed Skeletal Muscle 120 (May)

Immunology

Polyneuropathy, Organomegaly. Endocrinopathy, Monoclonal Gammopathy and Skin Changes (Poems) Syndrome 60 (Feb)

Proliferating Cell Nuclear Antigen Expression in the Survival of Astrocytoma Patients 306 (Nov)

Infarction

NMDA Receptor Blockade and Spinal Cord Ischemia Due to Aortic Crossclamping in the Rat Model 227 (Aug)

Infectious Diseases

Traitement Medico-chirurgical du Mal de Pott de L'adulte. Notre Attitude au Gabon 339 (Nov)

Intensive Care

Intensive Care of Acute Guillain-Barré Syndrome S23 (Suppl 1)

Assessment of Respiratory Function in the Intensive Care Unit S28 (Suppl 1)

Intracranial Pressure

Somatosensory Evoked Potential and Intracranial Pressure in Severe Head Injury 219 (Aug)

In Memoriam

George Gordon Habib 287 (Aug)

Ischemic Brain Injury

NMDA Receptor Blockade and Spinal Cord Ischemia Due to Aortic Crossclamping in the Rat Model 227 (Aug)

Magnetic Resonance Imaging

Acute Transverse Myelitis: A Retrospective Study Using Magnetic Resonance Imaging 133 (May)

A Controlled Trial of Mitozantrone in Multiple Sclerosis: Serial MRI Evaluation at One Year 266 (Aug)

Striatonigral Degeneration: Iron Deposition in Putamen Correlates with the Slit-like Void Signal of Magnetic Resonance Imaging 311 (Nov)

Frameless Stereotaxy for Pre-treatment Planning and Post-treatment Evaluation of Radiosurgery 319 (Nov)

Diagnosis of Vascular Dementia: Consortium of Canadian Centres for Clinical Cognitive Research Consensus Statement 358 (Nov)

Memory

Hippocampal Stimulation of Fornical-lesioned Rats Improves Working Memory 100 (May)

Meningitis

Unilateral Creutzfeldt-Jakob Disease Presenting as Rapidly Progressive Aphasia 350 (Nov)

Metastatic Tumours

Complications of First Craniotomy for Intra-Axial Brain Tumor 213 (Aug)

Mitoxantrone

A Controlled Trial of Mitozantrone in Multiple Sclerosis: Serial MRI Evaluation at One Year 266 (Aug)

Movement Disorders

Gilles de la Tourette Syndrome 48 (Feb)

Striatonigral Degeneration: Iron Deposition in Putamen Correlates with the Stit-like Void Signal of Magnetic Resonance lmaging 311 (Nov)

Multiple Sclerosis

The Impact of Fatigue on Patients with Multiple Sclerosis 9 (Feb)

Long-Term Results after Glycerol Rhizotomy for Multiple Sclerosis-Related Trigeminal Neuralgia 137 (May)

A Prevalence Study of Multiple Sclerosis in the Crowsnest Pass Region of Southern Alberta 262 (Aug)
A Controlled Trial of Mitozantrone in Multiple Sclerosis: Serial MRI Evaluation at One year 266 (Aug)

Intractable Hiccup due to Multiple Sclerosis: MR lmaging of Medullary Plaque 27 ( Aug)

Muscle

Mild Hypothermia Preserves Contractile Function and Inhibits Prostaglandin E2 Release from Metabolically Stressed Skeletal Muscle 120 (May)

Muscular Dystrophy

Autosomal Recessive. Fatal Infantile Hypertonic Muscular Dystrophy among Canadian Natives 203 (Aug)

Myasthenia Gravis

Usefulness of Single Fiber EMG for Distinguishing Neuromuscular from other Causes of Ocular Muscle Weakness I25 (May)

Myelopathy

Acute Transverse Myelitis: A Retrospective Study Using Magnetic Resonance Imaging 133 (May)

Myopathy

Mild Hypothermia Preserves Contractile Function and Inhibits Prostaglandin E2 Release from Metabolically Stressed Skeletal Muscle 120 (May)

Usefulness of Single Fiber EMG for Distinguishing Neuromuscular from other Causes of Ocular Muscle Weakness 125 (May)

Myelin

Autosomal Recessive Motor and Sensory Neuropathy with Excessive Myelin Ouffolding in Two Siblings 29 (Feb)

Nerve Biopsy

Complications of Sural Nerve Biopsy in Diabetic verus Non-Diabetic Patients 34 (Feb)

Nerve Conduction

Role of Electrophysiological Studies in Diabetic Neuropathy S8 (Suppl 4)

Neuro-Oncology

Unusual Evolution and Computerized Tomographic Appearance of a Gliosarcoma 141 (May)

Complications of First Craniotomy for Intra-Axial Brain Tumor 213 (Aug)

NMDA Receptor Blockade and Spinal Cord Ischemia Due to Aortic Crossclamping in the Rat Model 227 (Aug)

Malignant Rhabdoid Tumour of the Pineal Region 273 (Aug)

Proliferating Cell Nuclear Antigen Expression in the Survival of Astrocytoma Patients 306 (Nov)

Neuro-Ophthalmology

Adaptive Control of Eye Movements: Clinical Implications 177 (Aug)

Neurocritical Care

Neurocritical Care Precis of Panel Discussion S3 (Suppl 1)

Planning Care for Neurology and Neurosurgery Patients with Critical lllnesses 295 (Nov)

Neurocritical Care Symposium Supplement SI (Suppl 1)

Neuroendocrinology

Polyneuropathy, Organomegaly, Endocrinopathy Monoclonal Gammopathy and Skin Changes (Poems) Syndrome 60 (Feb)

Neurofibroma

Newborn Apnea Caused by a Neurofibroma at the Craniocervical Junction 64 (Feb)

Neuroimmunology

Iatrogenic False Aneurysm Following Repair of Intracranial Aneurysm 346 (Nov) 
Neurology - Paediatric

The Place of Neuronal Migration Abnormalities in Child Neurology 185 (Aug)

Neuronal Migration

The Place of Neuronal Migration Abnormalities in Child Neurology 185 (Aug)

\section{Neuropathology}

Fatal Rebleeding from a Dural Arteriovenous Malformation of the Posterior Fossa: case repor with pathological examination 67 (Feb)

Alcoholic Dementia: A Critical Review 88 (May)

Striatonigral Degeneration: Iron Deposition in Putamen Correlates with the Slit-like Void Signal of Magnetic Resonance Imaging 311 (Nov)

Pathological Definition and Evaluation of Diabetic Neuropathy and Clinical Correlations S13 (Suppl 4)

Neuropathology - Pediatric

Acute and Chronic Cerebral White Matter Damage in Neonatal Hydrocephalus 299 (Nov)

Neuropeptides

A Segmental Chronic Pain Syndrome in Rats Associated with Intrathecal Infusion on NMDA: evidence for selective action in the dorsal horn 24 (Feb)

Neuropharmacology

The Effect of Anticonvulsant Drugs on GABAStimulated Chloride Uptake 3 (Feb)

Antiepileptic Drug Selection and Adverse Effects: An Overview S3 (Suppl 3)

Acute and Chronic Toxicity of Antiepileptic Medications: a Selective Review S7 (Suppl 3)

Cognitive Side Effects of Antiepileptic Drugs S12 (Suppl 3)

Neurophysiology

Large Unresponsive Zones Appear in Cat Somatosensory Contex Immediately After Ulnar Nerve Cut 233 (Aug)

\section{Neuropsychology}

Neuropsychological Functioning in a Patient with Unilateral Cerebellar Damage 353 (Nov)

Neurosurgery

Neurosurgery at the Toronto General Hospital, 1924 - 1990: Part 1146 (May)

Neurosurgery at the Toronto General Hospital. 1924 - 1990: Part 2278 (Aug)

Neurosurgery - Adult

Iatrogenic False Aneurysm Following Repair of Intracranial Aneurysm 346 (Nov)

Neurosurgery - Paediatric

Acute and Chronic Cerebral White Matter Damage in Neonatal Hydrocephalus 299 (Nov)

Oculopharyngeal

Usefulness of Single Fiber EMG for Distinguishing Neuromuscular from other Causes of Ocular Muscle Weakness 125 (May)

Pain

A Segmental Chronic Pain Syndrome in Rats Associated with Intrathecal Infusion on NMDA evidence for selective action in the dorsal horn 24 (Feb)

Long-Term Results after Glycerol Rhizotomy for Multiple Sclerosis-Related Trigeminal Neuralgia 137 (May)

Parkinson's Disease

Dysphagia in Ambulant Patients with Parkinson's Disease: common, not dangerous 53 (Feb)

Influence of Bolus Volume on Hyoid Movements in Normal Individuals and Patients with Parkinson's Disease 57 (Feb)

Fluoxetine and Selegiline - Lack of Significant Interaction 259 (Aug)
Peripheral Nerve

A Segmental Chronic Pain Syndrome in Rats Associated with Intrathecal Infusion on NMDA evidence for selective action in the dorsal horn 24 (Feb)

Autosomal Recessive Motor and Sensory Neuropathy with Excessive Myelin Ouffolding in Two Siblings 29 (Feb)

Large Unresponsive Zones Appears in Cat Somatosensory Cortex Immediately After Ulnar Nerve Cut 233 (Aug)

Peripheral Neuropathy

Complications of Sural Nerve Biopsy in Diabetic versus Non-Diabetic Patients 34 (Feb)

Femoral Neuropathy in Renal Transplantation 38 (Feb)

Polyneuropathy, Organomegaly, Endocrinopathy, Monoclonal Gammopathy and Skin Changes (Poems) Syndrome 60 (Feb)

The Influence of Sulindac on Experimental Streptozotocin-Induced Diabetic Neuropathy 194 (Aug)

Clinical Testing in Diabetic Neuropathy S3 (Suppl 4) Role of Electrophysiological Studies in Diabetic Neuropathy S8 (Suppl 4)

Pathological Definition and Evaluation of Diabetic Neuropathy and Clinical Correlations S13 (Suppl 4)

End-Stage Complications of Diabetic Neuropathy: Foot Ulceration SI8 (Suppl 4)

Update on Diabetic Neuropathy - Speakers' Panel S23 (Suppl 4)

Pineal Gland

Maligant Rhabdoid Tumour of the Pineal Region 273 (Aug)

Poems Syndrome

Polyneuropathy, Organomegaly, Endocrinopathy, Monoclonal Gammopathy and Skin Changes (Poems) Syndrome 60 (Feb)

Prostaglandin

Mild Hypothermia Preserves Contractile Function and Inhibits Prostaglandin E2 Release from Metabolically Stressed Skeletal Muscle 120 (May)

Radiosurgery

Frameless Stereotaxy for Pre-treatment Planning and Post-treatment Evaluation of Radiosurgery 319 (Nov)

Renal Disease

Femoral Neuropathy in Renal Transplantation 38 (Feb)

Respiratory

Assessment of Respiratory Function in the Intensive Care Unit S28 (Suppl 4)

Respirology

Newborn Apnea Caused by a Neurofibroma at the Craniocervial Junction 64 (Feb)

Rhabdoid Tumour

Malignat Rhabdoid Tumour of the Pineal Region 273 (Aug)

Richardson Lecture

Adaptive Control of Eye Movements: Clinical Implications 177 (Aug)

Selegiline

Fluoxetine and Selegiline - Lack of Significant Interaction 259 (Aug)

Single Fiber EMG

Usefulness of Single Fiber EMG for Distinguishing Neuromuscular from other Causes of Ocular Muscle Weakness 125 (May)

\section{Somatosensory Cortex}

Large Unresponsive Zones Appears in Cat Somatosensory Cortex Immediately After Ulnar Nerve Cut 233 (Aug)

Somatosensory Evoked Potential

Somatosensory Evoked Potential and Intracranial Pressure in Severe Head Injury 219 (Aug)

Spinal Cord

Adult Diastematomyelia 72 (Feb)

NMDA Receptor Blockade and Spinal Cord Ischernia Due to Aortic Crossclamping in the Rat Model 227 (Aug)

Spinal Cord

Spinal Somatosensory Evoked Potentials in Patients with Tethered Cord Syndrome 325 (Nov)

Traitement Medico-chirurgical du Mal de Pott de L'adulte. Notre Attitude au Gabon 339 (Nov)

Stereotactic

Frameless Stereotaxy for Pre-treatment Planning and Post-treatment Evaluation of Radiosurgery 319 (Nov)

Streptozotocin

The Influence of Sulindac on Experimental Streptozotocin-Induced Diabetic Neuropathy 194 (Aug)

Striatonigral Degeneration

Striatonigral Degeneration: Iron Deposition in Putamen Correlates with the Slit-like Void Signal of Magnetic Resonance Imaging 311 (Nov)

Stroke

Parieto-Cerebellar Loop Impairment in Ataxic Hemiparesis: Proposed Pathophysiology Based on an Analysis of Cerebral Blood Flow 15 (Feb) Sulindac

The Influence of Sulindac on Experimental Streptozotocin-Induced Diabetic Neuropathy 194 (Aug)

Swallowing

Dysphagia in Ambulant Patients with Parkinson's Disease: common, not dangerous 53 (Feb)

Influence of Bolus Volume on Hyoid Movements in Normal Individuals and Patients with Parkinson's Disease 57 (Feb)

Newbom Apnea Caused by a Neurofibroma at the Craniocervical Junction 64 (Feb)

Tics

Gilles de la Tourette Syndrome 48 (Feb)

Toronto General Hospital

Neurosurgery at the Toronto General Hospital, 1924 - 1990: Part I 146 (May)

Neurosurgery at the Toronto General Hospital, 1924 - 1990: Part 2278 (Aug)

Toxicology

Acute and Chronic Toxicity of Antiepileptic Medications: a Selective Review S7 (Suppl 3)

Transplantation

Femoral Neuropathy in Renal Transplantation 38 (Feb)

Trigeminal Neuralgia

Long-Term Results after Glycerol Rhizotomy for Multiple Sclerosis-Related Trigeminal Neuralgia 137 (May)

Tuberculosis

Traitement Medico-chirurgical du Mal de Pott de L'adulte. Notre Attitude au Gabon 339 (Nov)

Vestibular

Adaptive Control of Eye Movements: Clinical Implications 177 (Aug)

Wernicke-Korsakoff Syndrome

Alcoholic Dementia: A Critical Review 88 (May) 